\title{
A Novel Prediction Process of the Remaining Useful Life of Electric Vehicle Battery Using Real-World Data
}

\author{
Xu Wang ${ }^{1}$, Jian Li $^{1}{ }^{\oplus}$, Ben-Chang Shia ${ }^{1,2}{ }^{\infty}$, Yi-Wei Kao ${ }^{1,2}\left(\right.$, Chieh-Wen Ho ${ }^{2,3}$ and Mingchih Chen ${ }^{1,2, *}$ \\ 1 Graduate School of Business Administration, Fu Jen Catholic University, No. 510, Zhongzhen Road, \\ Xinzhuang District, New Taipei City 24205, Taiwan; 406088165@mail.fju.edu.tw (X.W.); \\ lijian.fju@gmail.com (J.L.); 025674@mail.fju.edu.tw (B.-C.S.); kyw498762030@gmail.com (Y.-W.K.) \\ 2 Artificial Intelligence Development Center, Fu Jen Catholic University, No. 510, Zhongzhen Road, \\ Xinzhuang District, New Taipei City 24205, Taiwan; cwho1220@gmail.com \\ 3 Department of Life Science, National Taiwan University, Taipei 10617, Taiwan \\ * Correspondence: 081438@mail.fju.edu.tw
}

Citation: Wang, X.; Li, J.; Shia, B.-C.; Kao, Y.-W.; Ho, C.-W.; Chen, M. A

Novel Prediction Process of the Remaining Useful Life of Electric Vehicle Battery Using Real-World Data. Processes 2021, 9, 2174.

https://doi.org/10.3390/pr9122174

Academic Editor: Chih-Te Yang and Chi-Jie Lu

Received: 1 October 2021

Accepted: 26 November 2021

Published: 2 December 2021

Publisher's Note: MDPI stays neutral with regard to jurisdictional claims in published maps and institutional affiliations.

Copyright: () 2021 by the authors. Licensee MDPI, Basel, Switzerland. This article is an open access article distributed under the terms and conditions of the Creative Commons Attribution (CC BY) license (https:// creativecommons.org/licenses/by/ $4.0 /)$.
Abstract: In modern society, environmental sustainability is always a top priority, and thus electric vehicles (EVs) equipped with lithium-ion batteries are becoming more and more popular. As a key component of EVs, the remaining useful life of battery directly affects the demand of the EV supply chain. Accurate prediction of the remaining useful life (RUL) benefits not only EV users but also the battery inventory management. There are many existing methods to predict RUL based on state of health $(\mathrm{SOH})$, but few of them are suitable for real-world data. There are several difficulties: (1) battery capacity is not easy to obtain in the real world; (2) most of these methods use the individual data for each battery, and the computing processes are difficult to perform in the cloud; (3) there is a lack of approaches for real-time SOH estimating and RUL predicting. This paper adopts several statistical methods to perform the prediction and compars the results of different models on experimental data (NASA dataset). Then, real-world data were implemented for an online process of RUL prediction. The main finding of this research is that the required CPU time was short enough to meet the daily usage after the real-world data was implemented for an online process of RUL prediction. The feasibility and precision of the prediction model can help to support the frequency control in power systems.

Keywords: big data analysis; remaining useful life; Lasso regression; ARIMA; Monte-Carlo simulation

\section{Introduction}

Rechargeable lithium-ion batteries have been widely used in applications ranging from portable electronics to EVs in modern life due to their many advantages, such as their high volumetric and gravimetric energy density and low self-discharge rate [1]. Therefore, the growth of the EV market has been very rapid.

The safety and reliability of EVs compared to those of traditional vehicles are the top concerns of EV users. However, both safety and reliability are subject to not only the battery technology but also the management system for the battery [2]. The battery management system (BMS) has become one of the chief components in EVs. It provides the capability to monitor the working status of the battery and maintain all the cells within their operating limits. In particular, a BMS is expected to provide information about the three critical characteristics of a battery, namely the state of charge (SOC), the state of health (SOH), and the remaining useful life (RUL) [3].

The SOC is a measure of the remaining capacity of the battery. Due to the inherent chemical reactions of a battery, it is difficult to obtain a fully accurate value. There are two approaches to determining the SOC. The first is the direct approach, such as coulomb counting, which simply measures the remaining capacity by using the current integration [4]. 
On the other hand, indirect methods usually investigate the relationship between the SOC and some other electrical parameters such as open-circuit voltage (OCV) and impedance [5].

Traditional approaches to battery health management have mostly focused on SOC problems. However, in recent years, attention has increasingly been paid to the $\mathrm{SOH}$ and RUL. The $\mathrm{SOH}$ is a measure of a battery's capability to deliver its specified output. Unlike the SOC, there is no clear-cut definition of the $\mathrm{SOH}$ [2].

The RUL refers to the available service time left before a system degrades to an unacceptable level [6], which is the EOL of the battery. It has also been suggested that when using an EV battery, an indicator $S O H_{E O L}$ is $0 \%$ when the battery capacity decreases to a certain level $(70 \%$ or $80 \%$ of the nominal capacity is often considered to be the failure threshold) [7]. Below is the equation.

$$
S O H_{E O L}=\frac{Q_{a c t}-Q_{\text {threshold }}}{Q_{R}-Q_{\text {threshold }}} \times 100 \%
$$

where $Q_{R}$ is the rated capacity and $Q_{a c t}$ is the actual capacity of the battery that is degraded. $Q_{\text {threshold }}$ is the capacity of the failure threshold. Therefore, the RUL indicates the remaining time or the number of load cycles until the battery reaches an $\mathrm{SOH}_{E O L}$ of $0 \%$.

A general definition of $\mathrm{SOH}$ is that it reflects the performance of a battery relative to its fresh condition. Therefore, the $\mathrm{SOH}$ is defined as $100 \%$ for a fresh cell and $0 \%$ for a cell that has reached the limitations of the end of life (EOL) [8]. Typically, the following equation in percentage form is used:

$$
S O H=\frac{Q_{a c t}}{Q_{R}} \times 100 \%
$$

We note that the capacity, which quantifies the available energy stored in a fully charged Li-ion battery cell, is the indicator used most frequently to measure the $\mathrm{SOH}$. However, it is not the only one. For example, Zhou et al. [9] proposed a novel health indicator that is formed by a series of battery discharging voltages.

Most previous works in RUL prediction used Equation (2) to measure $\mathrm{SOH}$ such as the NASA dataset we use in the following sections. We therefore use Equation (2) in this paper and employ a failure threshold of $70 \%$.

Figure 1 shows the relationship between the critical characteristics of a battery. The raw data from BMSs can be used to estimate the parameters or create models for specific purposes. Various studies focus on estimating the capacity and use it to estimate the SOC and $\mathrm{SOH}$. When the $\mathrm{SOH}$ is determined, there are many methods of computing the probability density function (PDF) of the EOL so that the RUL can be predicted. Moreover, this paper introduces some real-world parameters such as driving behavior and environmental information that supplement $\mathrm{SOH}$ estimation and RUL prediction.

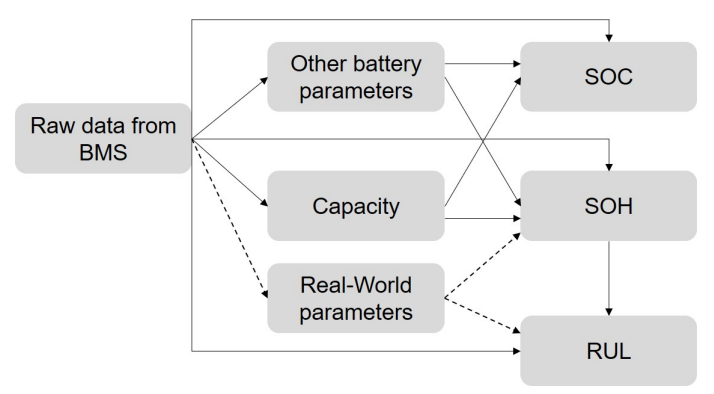

Figure 1. Relationships among the characteristics of a battery.

Barré et al. [10] divided the existing models for battery RUL prediction into five types: (1) electrochemical models, (2) equivalent circuit-based models, (3) performancebased models, (4) analytical models with empirical fitting, and (5) statistical approaches. Zhao et al. [11] simplified them into three parts: (1) electrochemical models, which need 
to use physical equations and are usually complex; (2) equivalent circuit-based models, such as particle filtering (PF) and other filtering methods; and (3) statistical models, which use data-driven methods without needing any prior knowledge about the battery aging mechanisms.

It is difficult to use physical equations and electrochemical reactions to obtain a reliable model. Most recent studies have focused on the last two types, namely model-based methods and data-driven methods. Model-based methods can be divided into two types: empirical equations and electrochemical principles [12]. Data-driven methods can be divided into two categories according to type of data. One is direct condition monitoring data, which are the data that can describe the underlying state of the system directly. We can use regression-based models, Wiener processes, gamma processes, and Markovianbased models to deal with them. On the other hand, for models based on indirect condition monitoring data, we include stochastic filtering-based models, covariate-based hazard models, hidden Markov models (HMMs), and hidden semi-Markov models (HSMMs) [13].

Zheng and Fang [14] indicated that the approaches that are purely model-based filtering and those that are purely data-driven have their respective limitations. They proposed hybrid approaches that incorporate model-based filtering approaches and datadriven approaches and classified them into three types: (1) models that compensate for the physical state/measurement model, (2) approaches to predict future trends in measurement values, and (3) methods to estimate the model parameters for the physical-based methods to predict.

Rather than data-driven methods, the model-based filtering techniques that feature closed-loop expressions can self-correct and overcome unexpected disturbances [15]. A Kalman filter-based prognostic method was developed to predict the battery RUL in 2009 [16]. Dong et al. [17] improved the standard particle filter and developed an SVR-PF that introduced novel capacity degradation parameters to determine the battery health in real time in 2014. Zhang et al. [18] proposed an improved unscented particle filtering (IUPF) method for lithium-ion battery RUL prediction based on the Markov chain Monte Carlo (MCMC) method in 2017. Duong and Raghavan [19] introduced a Heuristic Kalman algorithm in 2018, a metaheuristic optimization approach, that was combined with particle filtering to tackle sample degeneracy. Zhang et al. [20] presented an improved PF algorithm in 2018 based on linear optimizing combination resampling (U-LOCR-PF) to improve prediction accuracy. Ma et al. [21] developed a Gauss-Hermite particle filter (GHPF) to update the parameters of the capacity degradation model in real time and predict the RUL in 2019. Li et al. [22] developed an inheritance PF by using the genetic algorithm framework in 2019.

Data-driven methods can capture the inherent relationships and learn the trends present in the data to provide RUL predictions. They do not require specific knowledge of material properties, constructions, or failure mechanisms, and they do not involve the development of high-level physical models of the system; therefore, they have become increasingly popular for Li-ion battery RUL prediction [23]. Ng et al. [24] proposed a naive Bayes (NB) model for the RUL prediction of batteries under different operating conditions in 2014. Patil et al. [25] transformed the RUL into a classification problem in 2015 so that some machine learning methods could be used to estimate the RUL level. Zhou and Huang [26] proposed a novel approach that combines empirical mode decomposition (EMD) and the autoregressive integrated moving average (ARIMA) model in 2016. Zhang et al. [27] developed an RUL-prediction method in 2019 based on the Box-Cox transformation (BCT) and Monte Carlo (MC) simulation.

In recent years, deep learning has become very popular in all fields. Wu et al. [28] investigated the relationship between the RUL and the charge curve and simulated it in 2016 using a feed-forward neural network (FFNN) due to its simplicity and effectiveness. Zhang et al. [29] employed the long short-term memory (LSTM) recurrent neural network (RNN) in 2018 to learn the long-term dependencies among the degraded capacities of 
lithium-ion batteries. Khumprom and Yodo [30] presented a deep neural network (DNN) approach in 2019 to predict the SOH and the RUL.

In addition to the general parameters extracted from monitoring data, some other methods deserve attention. Chen et al. [31] presented a geometrical approach to Li-ion battery capacity estimation in 2014. They utilized four geometrical features that are sensitive to slight changes and degradation in performance. Wang et al. [32] found that different discharge rates (DDRs) affect both usable battery capacity and battery degradation rates. They designed an experiment to collect accelerated battery life testing data for DDRs in 2017, which were used to investigate how DDRs influence usable battery capacity.

Besides the aging of batteries, calendar aging (i.e., aging at rest) is also very important since parking time can reach $90 \%$ of the total vehicle lifetime. Eddahech et al. [33] presented a method for the calendar aging quantification of power batteries taking into account the SOC and temperature effects.

In the real world, the relation between battery parameters and the RUL is more complicated. The condition of a Li-ion battery is affected by the road conditions, environmental temperatures, charge modes, and even the driver's behavior. Nuhic et al. [34] used realword data to estimate $\mathrm{SOH}$ and RUL but lack applicable data and thus are based on uniform tests. Canals et al. [35] extracted temperature, voltage, current, and energy exchanges data from on-board data-loggers installed in an EV and calculated the internal resistance and voltage recovery, so that the $\mathrm{SOH}$ can be estimated.

However, few of them are suitable for real-world data. There are several difficulties: (1) battery capacity is not easy to obtain in the real world; (2) most of these methods use individual data for each battery, and the computing processes are difficult perform in the cloud; (3) there is a lack of approaches for real-time SOH estimating and RUL prediction.

Many studies mentioned above focused on creating models for batteries separately. Indeed, these are accurate approaches if data are sufficient. Most automobiles do not have enough computing power to improve computing speed and save resources. This paper proposes to create an aggregated model for all of the vehicle data and use personalization parameters to reflect the individual characteristics.

This paper attempts to introduce more influencing factors for RUL prediction based on real-world data and proposes an online data-driven approach to rapidly estimating the $\mathrm{SOH}$ and RUL. The required CPU time was short enough to meet the daily usage after the real-world data were implemented for an online process of RUL prediction. Alhelou et al. [36] discussed the contribution of EVs in supporting the frequency control in power systems, and the feasibility and precision of the RUL prediction model of EVs can help to overcome the uncertainties of the electric demands.

\section{Materials and Methods}

\subsection{Overview}

Figure 2 shows the process structure of RUL prediction based on real-world data. The raw data usually come from a BMS and do not contain accurate $\mathrm{SOH}$ value, so we need to calculate rough $\mathrm{SOH}$ at first and then create a statistical model using real-world parameters. When we successfully forecast or simulate the future parameters, this statistical model could be used to predict the future $\mathrm{SOH}$ and RUL.

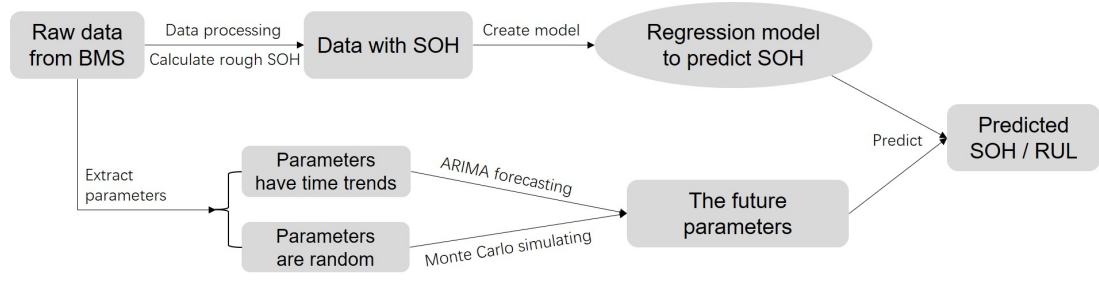

Figure 2. Process of RUL prediction based on real-world data. 
In this study, we first use the NASA Ames Li-ion battery cycle life test data [37] since they contain accurate capacity values, allowing us to train precise models and compare the methods. Figure 3 shows the process using NASA data.

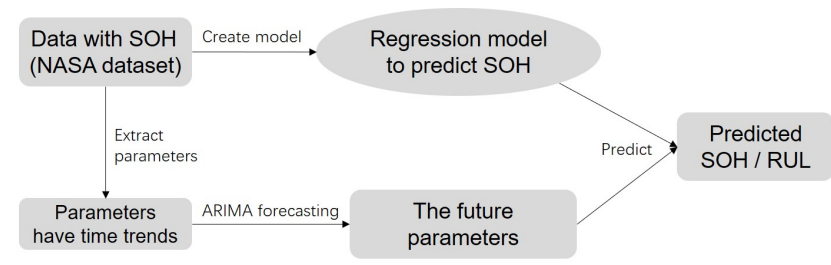

Figure 3. Process of RUL prediction based on NASA data.

\subsection{Introduction to NASA Dataset}

There are four Li-ion batteries (\#5, \#6, \#7, and \#18), which were run through 3 different operational profiles (charge, discharge, and impedance) at room temperature. Charging was carried out in constant current (CC) mode at $1.5 \mathrm{~A}$ until the battery voltage reached $4.2 \mathrm{~V}$ and then continued in a constant voltage (CV) mode until the charge current dropped to $20 \mathrm{~mA}$. Discharge was carried out at a constant current (CC) level of $2 \mathrm{~A}$ until the battery voltage fell to $2.7 \mathrm{~V}, 2.5 \mathrm{~V}, 2.2 \mathrm{~V}$, and $2.5 \mathrm{~V}$ for batteries \#5, \#6, \#7, and \#18, respectively. The experiments were stopped when the batteries reached the EOL criterion, which was a $30 \%$ fade in rated capacity (from $2 \mathrm{Ahr}$ to $1.4 \mathrm{Ahr}$ ). Figure 4 shows the relationship between the capacity and cycle number. The number of cycles are 168, 168, 168, 132, for batteries $\# 5, \# 6, \# 7$, and \#18.
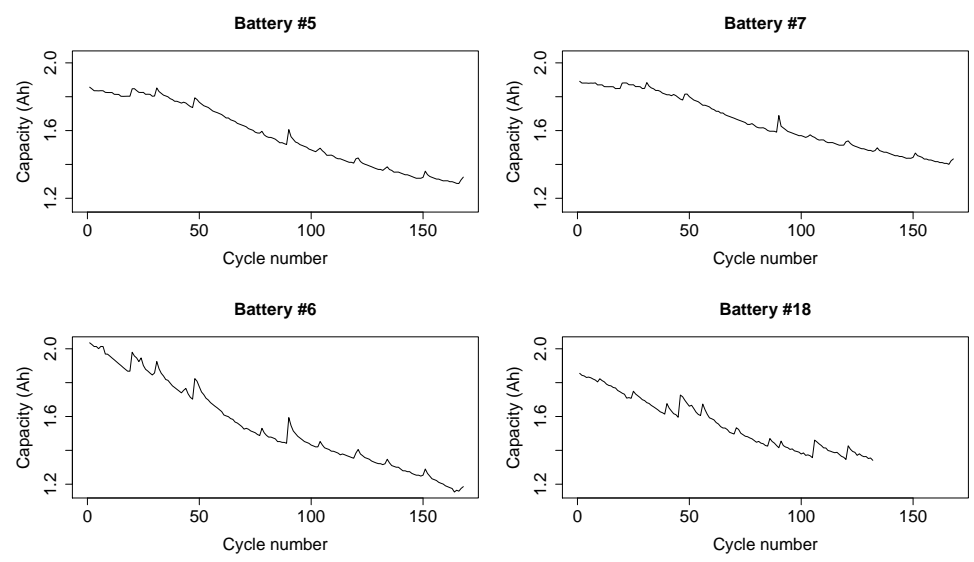

Figure 4. Plots of the data: battery capacity based on the cycle number.

According to Equation (2), we can compute the $\mathrm{SOH}$ value at each point, and the results are shown in Figure 5.
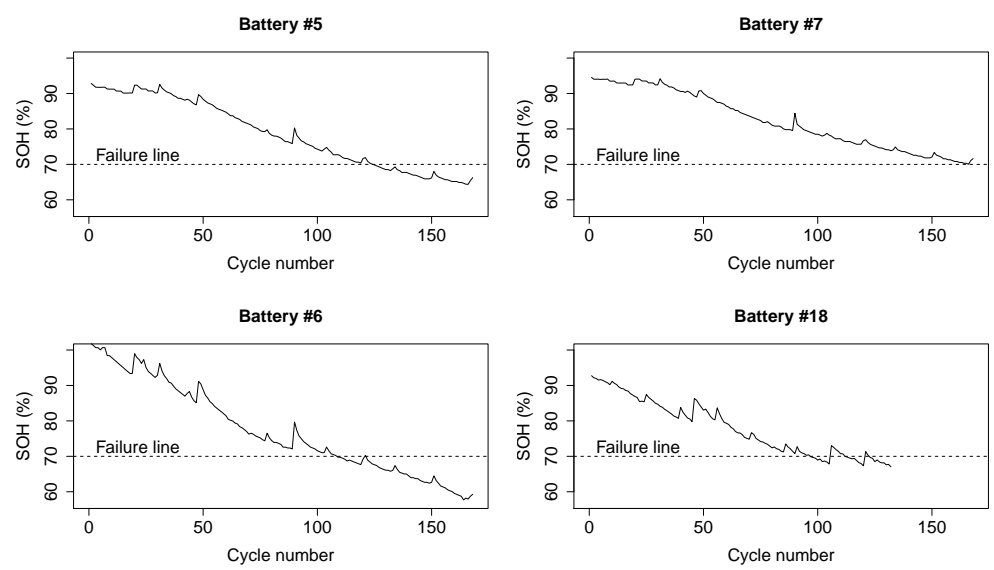

Figure 5. Plots of the data: $\mathrm{SOH}$ based on the cycle number. 
Table 1 shows some sample records of NASA dataset.

Table 1. Sample of NASA dataset.

\begin{tabular}{|c|c|c|c|c|c|c|c|c|c|}
\hline Type & Time_Start & Time_End & Capacity & $v_{1}$ & $\cdots$ & $\overline{i_{1}}$ & $\cdots$ & $t_{1}$ & $\ldots$ \\
\hline charge & $2008 / 4 / 2$ 13:08 & $2008 / 4 / 2$ 15:14 & & 4.187 & & 0.643 & & 25.32 & \\
\hline discharge & $2008 / 4 / 215: 25$ & $2008 / 4 / 216: 27$ & 1.856487 & 3.530 & & -1.819 & & 32.57 & \\
\hline charge & $2008 / 4 / 216: 37$ & $2008 / 4 / 2$ 19:33 & & 4.059 & & 0.949 & & 26.64 & \\
\hline discharge & 2008/4/2 19:43 & 2008/4/2 20:45 & 1.846327 & 3.537 & & -1.818 & & 32.73 & \\
\hline charge & $2008 / 4 / 2$ 20:55 & $2008 / 4 / 2$ 23:50 & & 4.058 & & 0.951 & & 26.78 & \\
\hline discharge & $2008 / 4 / 3$ 0:01 & 2008/4/3 1:01 & 1.835349 & 3.544 & & -1.816 & & 32.64 & \\
\hline
\end{tabular}

$v_{i}, i_{i}$, and $t_{i}$ mean the voltage, output current, and temperature of a battery at the $i$-th time point, respectively.

\subsection{SOH Estimation}

The NASA dataset contains an accurate capacity in each discharge cycle; therefore, we can calculate the accurate $\mathrm{SOH}$ and use it as the response variable. We extracted some features from the internal battery parameters (voltage, output current, and temperature) in each discharge cycle. Table 2 shows the variables in the dataset. There is only one realworld parameter: ambient temperature. In this dataset, the ambient temperature remains unchanged, so it will not be selected in the final models.

Table 2. Variables in each discharge cycle.

\begin{tabular}{llll}
\hline Variable & Description & Variable & Description \\
\hline soh & SOH in each discharge cycle & imin & minimum of output current \\
cycle & cycle number & iavg & average of output current \\
tambient & ambient temperature & isd & standard deviation of output current \\
vmax & maximum of voltage & tmax & maximum of temperature \\
vmin & minimum of voltage & tmin & minimum of temperature \\
vavg & average of voltage & tavg & average of temperature \\
vsd & standard deviation of voltage & tsd & standard deviation of temperature \\
imax & maximum of output current & & \\
\hline
\end{tabular}

We will try some regression models to predict $\mathrm{SOH}$. The voltage, output current, and temperature measurements in each discharge cycle were selected as independent variables. To obtain more features, we conducted feature engineering by extracting the maximum, minimum, mean, and standard deviation from all of the measurements. We defined these 3 models according to different data scenarios.

- Model 1: Use the individual data for each battery to predict that battery's SOH.

- Model 2: Use all of the data to create an aggregate model to predict each battery.

- Model 3: Use the data for the other batteries to predict the SOH for another battery. For example, we combine the data for batteries \#5, \#6, and \#7 to predict battery \#18.

Linear regression is the simplest model, but there may be correlation and linear dependence between explanatory variables. We will also therefore try Lasso and ridge regression.

The Lasso [38] (least absolute shrinkage and selection operator) is a regression with an $\ell_{1}$-norm penalty, which minimizes the residual sum of squares subject to the sum of the absolute value. Because of its good performance in both variable selection and prediction accuracy, Lasso is popular for predicting continuous variables. Mansouri et al. [39] discussed how to use Lasso to predict the RUL of a battery. To improve computing efficiency, Friedman et al. [40] developed fast algorithms to estimate generalized linear models with convex penalties. 
A general form of the Lasso is elastic net. Suppose that we have observations $x_{i} \in \mathbb{R}^{p}$ and the responses $y_{i} \in \mathbb{R}, i=1, \ldots, N$. The objective function is

$$
\min _{\left(\beta_{0}, \beta\right) \in \mathbb{R}^{p+1}} \frac{1}{2 N} \sum_{i=1}^{N}\left(y_{i}-\beta_{0}-x_{i}^{T} \beta\right)^{2}+\lambda\left[(1-\alpha)\|\beta\|_{2}^{2} / 2+\alpha\|\beta\|_{1}\right],
$$

where $\lambda \geq 0$ is a complexity parameter and $0 \leq \alpha \leq 1$ is a compromise between the $\ell_{1}$-norm and $\ell_{2}$-norm. $\alpha=0$ means the ridge regression, and $\alpha=1$ means the Lasso. The elastic net is a regularized regression method that linearly combines the $\ell_{1}$-norm and $\ell_{2}$-norm penalties of the Lasso and ridge methods.

We use the root mean square error (RMSE) to compare the performance of the methods:

$$
R M S E=\sqrt{\sum_{k=1}^{m}\left(\widehat{S O H}_{k}-S O H_{k}\right)^{2} / m}
$$

where $\mathrm{SOH}_{k}$ is the $\mathrm{SOH}$ value at cycle number $k$, and $\widehat{\mathrm{SOH}}$ represents the predicted value.

This approach is based on the internal battery parameters and predicts the current $\mathrm{SOH}$ value. It cannot be directly used to predict the future $\mathrm{SOH}$ and thus predict the RUL. Although there are many methods to estimate the SOH and predict the RUL at the same time, these methods usually have high requirements for data and have time-consuming calculation processes. In the real world, there are often missing values, and batteries are seldom fully discharged. Using this approach, we need only the internal battery parameters and other parameters of the current state to estimate the value of the current $\mathrm{SOH}$, which is meaningful for the residual value evaluation of the battery. In addition, the calculation process is both simple and fast, which is suitable for real-time computing on the cloud. Finally, the estimated $\mathrm{SOH}$ value can be combined with other methods, such as ARIMA, to predict the RUL.

\subsection{RUL Prediction}

\subsubsection{RUL Prediction by Using Time Series Method Directly}

A time series is a set of observations $x_{t}$, each one being recorded at a specified time $t$ [41]. Time series forecasting involves using existing data to make predictions about future events. The autoregressive integrated moving average (ARIMA) method is a popular model to identify complex patterns in data and to generate forecasts [42].

Hyndman and Khandakar [43] developed a step-wise algorithm for automatic forecasting with ARIMA models. By using this algorithm, we can select the model that minimizes the Akaike's information criterion (AIC) or Bayesian information criterion (BIC) among all of the models that are appropriate for the data.

The ARIMA method can be used to predict the RUL based on the historical data, and Zhou and Huang [26] have discussed the approaches to predict the battery RUL by forecasting the next step $\mathrm{SOH}$.

If we obtain historical $\mathrm{SOH}$ series, we can apply the ARIMA model to predict the future $\mathrm{SOH}$. First, we make the $\mathrm{SOH}$ prediction at 4 different starting points for each battery. For example, starting at point 60 means that we use data from cycle 1 to cycle 59 as the training dataset and then forecast the future values of the SOH. The RMSE is used as the evaluation criterion to evaluate the performance of $\mathrm{SOH}$ prediction.

\subsubsection{RUL Prediction by the Regression Methods}

When we use time series methods, a smaller number of data results in lower prediction accuracy. There is another strategy to determine the future parameters at first and then predict the $\mathrm{SOH}$, which is using regression methods.

If the parameters have time trends, we can use the ARIMA model to predict the future values. If the parameters are random or unpredictable, such as driving behaviours, we can 
use a Monte Carlo simulation to determine the future values. Then, regression models can be used to predict the $\mathrm{SOH}$.

If simulated data are used, we can obtain the PDF of the RUL. For the sake of simplicity, we use the ARIMA model to predict the internal battery parameters, and we then use the Lasso regression to predict the SOH and RUL.

\subsection{Results}

\subsubsection{SOH Estimation by Regression Models}

First, we employ a linear regression to predict the $\mathrm{SOH}$ for each battery. Figure 6 shows the prediction result of each model for each battery.
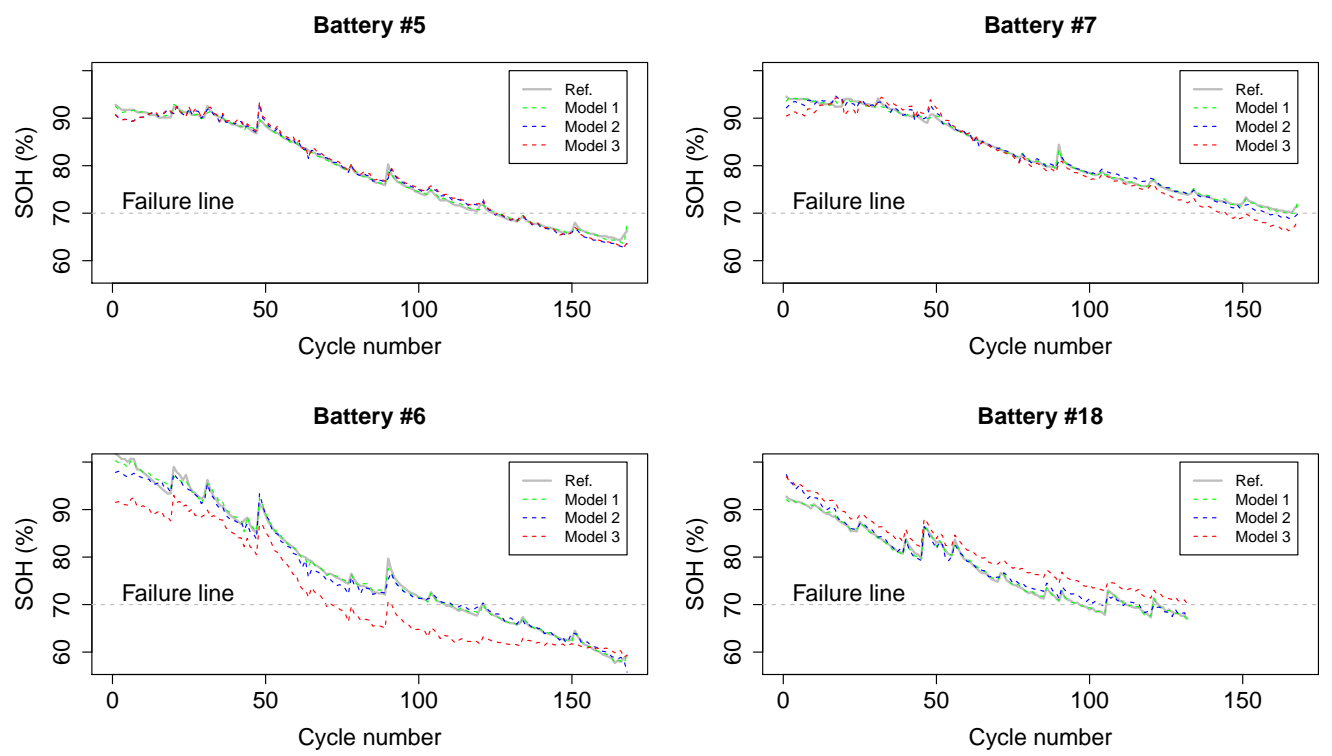

Figure 6. Prediction by using linear regression. 'Ref.' means the actual data.

We find that most of the results are good, except for model 3 on batteries \#6 and \#18.

Using the same definition of the 3 models, we employ a ridge regression to predict the $\mathrm{SOH}$. The results are shown in the Figure 7.

Model 3 on battery \#5 seems to obtain better performance, but the others are not as good as the linear regression.
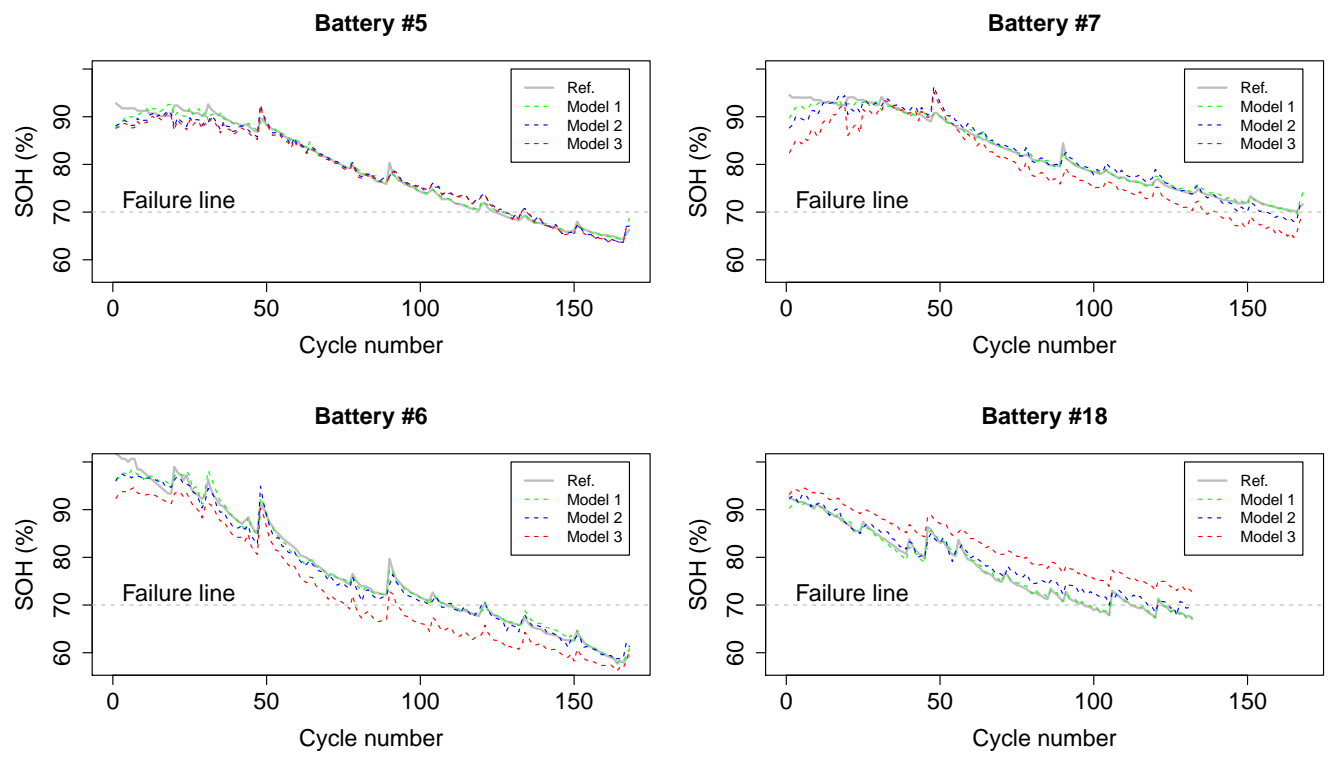

Figure 7. Prediction using a ridge regression. 'Ref.' means the actual data. 
Then, we employed the Lasso regression. The results are shown in the Figure 8.
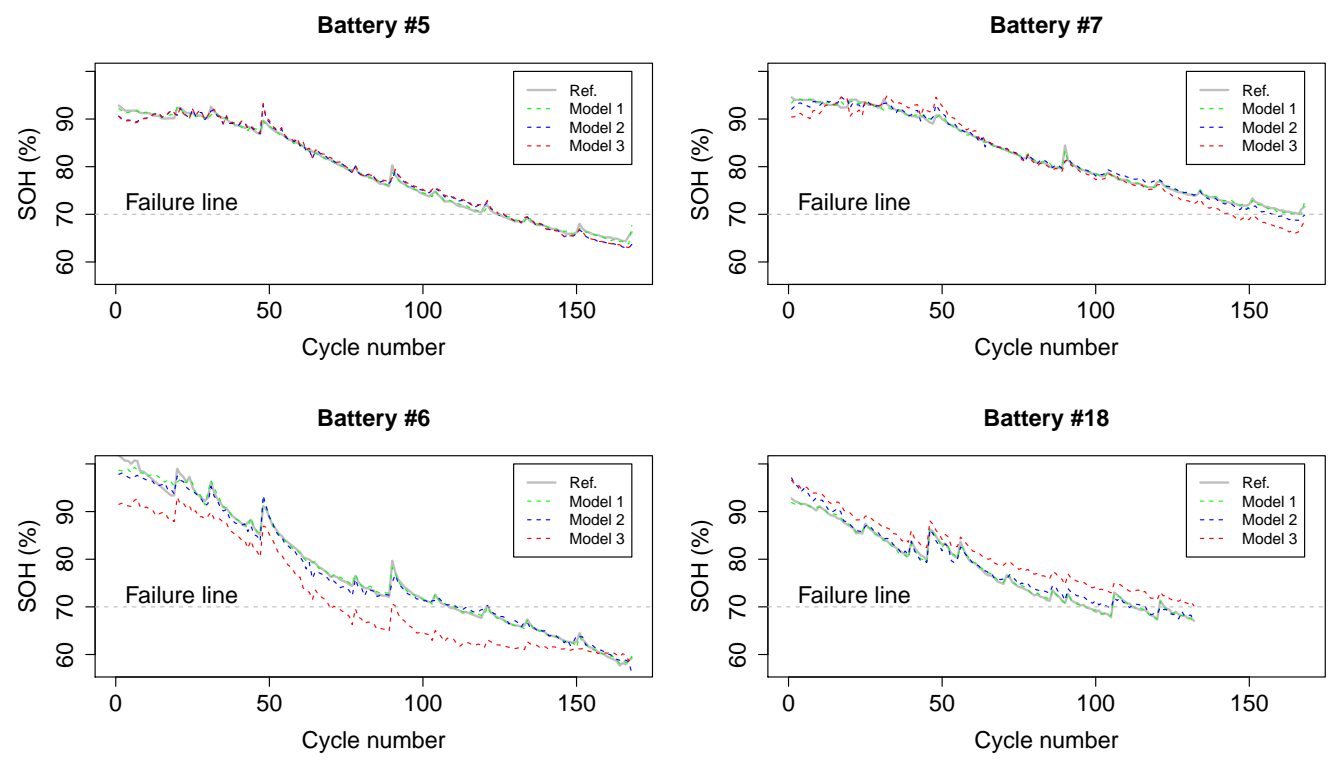

Figure 8. Prediction using the Lasso regression. 'Ref.' means the actual data.

Model 3 on battery \#6 seems to be a little bit better than the linear regression, and the others do not seem to be worse.

Table 3 shows the RMSE results of each method used for each battery, and we find that the linear regression model is the best in most cases. To prevent overfitting, we mainly refer to model 3 . We find that the ridge regression has the best prediction result for battery $\# 6$, but it has poor performance on the other batteries. The Lasso has similar performance as the linear regression.

Table 3. RMSE (\%) results of all the models.

\begin{tabular}{lccccccccc}
\hline \multirow{2}{*}{ Battery } & \multicolumn{3}{c}{ Linear Regression } & \multicolumn{3}{c}{ Ridge Regression } & \multicolumn{3}{c}{ Lasso Regression } \\
& Model 1 & Model 2 & Model 3 & Model 1 & Model 2 & Model 3 & Model 1 & Model 2 & Model 3 \\
\hline$\# 5$ & 0.4 & 0.93 & 1.04 & 0.91 & 1.42 & 1.6 & 0.41 & 0.94 & 1.02 \\
$\# 6$ & 0.63 & 1.22 & 5.57 & 1.09 & 1.45 & 4.44 & 0.77 & 1.2 & 5.64 \\
$\# 7$ & 0.39 & 0.84 & 1.87 & 0.85 & 1.68 & 3.78 & 0.41 & 0.88 & 1.98 \\
$\# 18$ & 0.32 & 1.24 & 3.2 & 0.55 & 1.72 & 4.95 & 0.33 & 1.23 & 3.33 \\
\hline
\end{tabular}

\subsubsection{RUL Prediction When $\mathrm{SOH}$ Is Determined}

In this paper, we set $70 \%$ of the $\mathrm{SOH}$ as the failure threshold. Thus, we can predict the RUL by counting the remaining life (in number of cycles) before the battery reaches the EOL. The absolute error (AE) is used to evaluate the performance of RUL prediction. The RMSE of $\mathrm{SOH}$ is computed according to Equation (4). Since the $\mathrm{SOH}$ prediction before the EOL is more important, we also list the RMSE when $\mathrm{SOH}>70 \%$. The results are shown in Figure 9 and Table 4.

Since the degradation of battery \#7 does not reach the failure threshold, the RMSE of the overall data is the same as the RMSE of the data when $\mathrm{SOH}>70 \%$. For the other batteries, the predictions are better when $\mathrm{SOH}>70 \%$. 

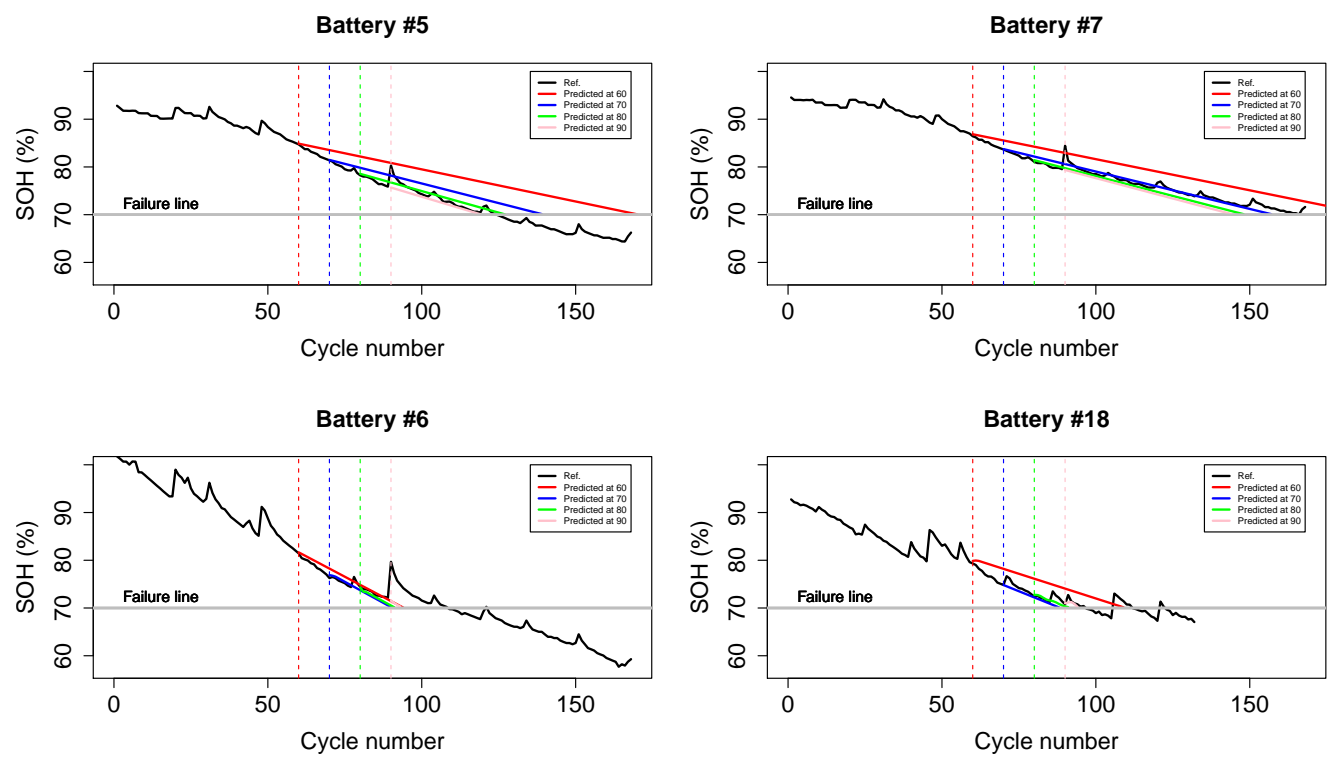

Figure 9. RUL prediction by ARIMA at different starting points. 'Ref.' means the actual data.

Table 4. Prediction results of ARIMA.

\begin{tabular}{|c|c|c|c|c|c|c|c|}
\hline Battery & $\begin{array}{l}\text { Starting } \\
\text { Point }\end{array}$ & $\begin{array}{l}\text { ARIMA } \\
(p, d, q)\end{array}$ & $\begin{array}{l}\text { Actual } \\
\text { RUL }\end{array}$ & $\begin{array}{l}\text { Predicted } \\
\text { RUL }\end{array}$ & $\mathrm{AE}$ & $\begin{array}{c}\text { RMSE of SOH \% } \\
\text { (Overall) }\end{array}$ & $\begin{array}{l}\text { RMSE of SOH \% } \\
(\text { SOH }>70 \%)\end{array}$ \\
\hline \multirow{4}{*}{$\# 5$} & 60 & $(0,1,0)$ & 65 & 111 & 46 & 5.32 & 4.44 \\
\hline & 70 & $(0,1,0)$ & 55 & 70 & 15 & 2.09 & 2.00 \\
\hline & 80 & $(0,1,0)$ & 45 & 48 & 3 & 1.01 & 0.92 \\
\hline & 90 & $(0,1,0)$ & 35 & 30 & 5 & 1.99 & 1.34 \\
\hline \multirow{4}{*}{$\# 6$} & 60 & $(0,1,0)$ & 49 & 35 & 14 & 7.67 & 3.05 \\
\hline & 70 & $(0,1,3)$ & 39 & 21 & 18 & 9.58 & 4.28 \\
\hline & 80 & $(0,1,3)$ & 29 & 12 & 17 & 9.65 & 4.64 \\
\hline & 90 & $(0,1,3)$ & 19 & 4 & 15 & 9.05 & 4.80 \\
\hline \multirow{4}{*}{$\# 7$} & 60 & $(0,1,0)$ & 109 & 129 & 20 & 2.90 & 2.90 \\
\hline & 70 & $(0,1,0)$ & 99 & 88 & 11 & 0.99 & 0.99 \\
\hline & 80 & $(0,1,0)$ & 89 & 69 & 20 & 1.84 & 1.84 \\
\hline & 90 & $(0,1,0)$ & 79 & 55 & 24 & 2.47 & 2.47 \\
\hline \multirow{4}{*}{$\# 18$} & 60 & $(0,1,3)$ & 37 & 50 & 13 & 2.43 & 2.73 \\
\hline & 70 & $(0,1,0)$ & 27 & 19 & 8 & 5.09 & 1.61 \\
\hline & 80 & $(2,1,3)$ & 17 & 12 & 5 & 4.68 & 1.23 \\
\hline & 90 & $(2,1,3)$ & 7 & 5 & 2 & 4.04 & 0.67 \\
\hline
\end{tabular}

\subsubsection{RUL Prediction When SOH Is Not Determined}

In the real world, battery capacity is not easy to obtain, so there is no accurate $\mathrm{SOH}$ value available when we predict the RUL. A feasible way would be forecasting the battery parameters at first and then predicting the RUL by regression model.

Firstly, we forecast the future parameters listed in Table 2 by the ARIMA model. Then, we use Model 2 of Linear/Ridge/Lasso regression to predict RUL. After comparing the results, we choose the Ridge regression model. The results are shown in Figure 10 and Table 5. 

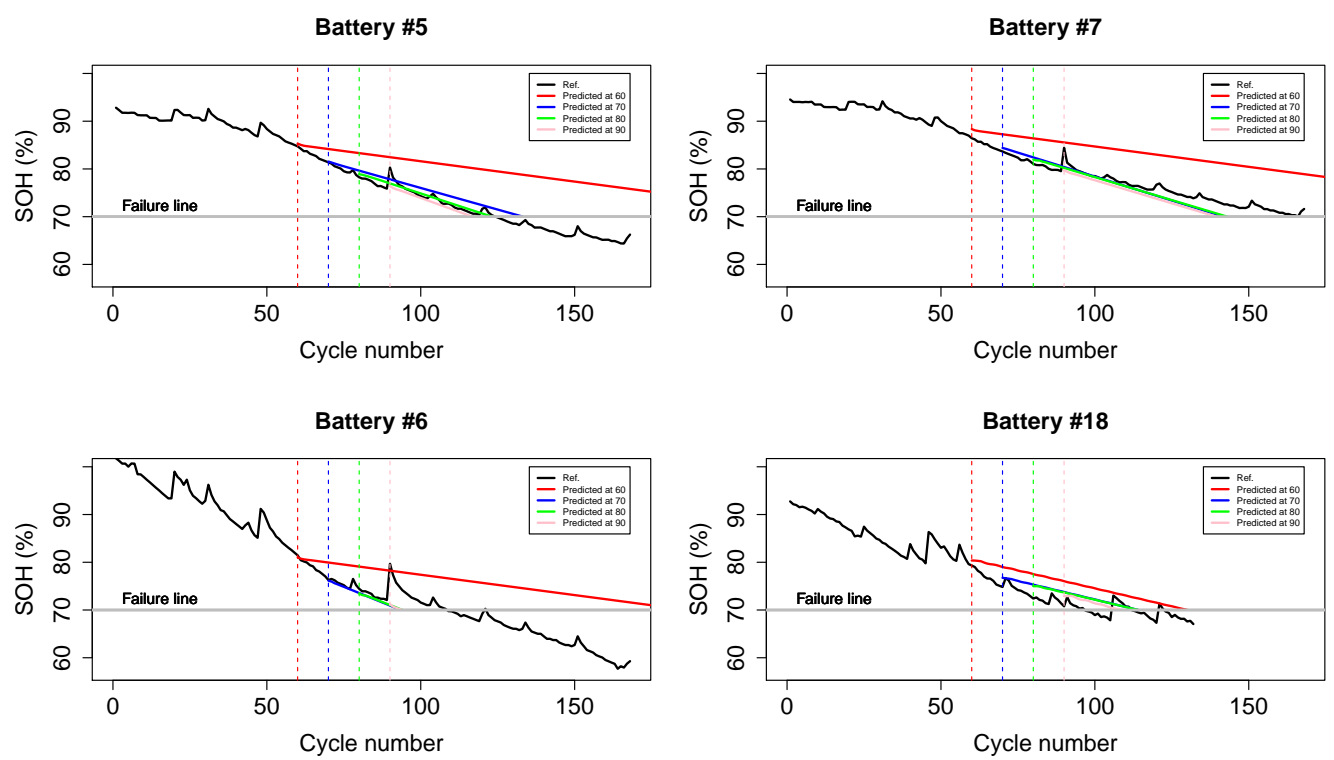

Figure 10. RUL prediction by the Ridge regression at different starting points. 'Ref.' means the actual data.

Table 5. Prediction results of regression models.

\begin{tabular}{cccccccc}
\hline Battery & $\begin{array}{c}\text { Starting } \\
\text { Point }\end{array}$ & $\begin{array}{c}\text { Actual } \\
\text { RUL }\end{array}$ & $\begin{array}{c}\text { AE } \\
\text { (Linear) }\end{array}$ & $\begin{array}{c}\text { AE } \\
\text { (Lasso) }\end{array}$ & $\begin{array}{c}\text { AE } \\
\text { (Ridge) }\end{array}$ & $\begin{array}{c}\text { RMSE \% } \\
\text { (Overall) }\end{array}$ & $\begin{array}{c}\text { RMSE \% } \\
\text { (SOH > 70\%) }\end{array}$ \\
\hline \multirow{4}{*}{ \#5 } & 60 & 65 & 85 & 84 & 112 & 8.44 & 6.37 \\
& 70 & 55 & 29 & 28 & 9 & 1.42 & 1.53 \\
& 80 & 45 & 35 & 33 & 1 & 1.70 & 0.87 \\
& 90 & 35 & 22 & 21 & 8 & 3.23 & 1.38 \\
\hline \multirow{4}{*}{ \#6 } & 60 & 49 & 54 & 54 & 78 & 7.84 & 4.44 \\
& 70 & 39 & 29 & 28 & 15 & 5.45 & 3.26 \\
& 80 & 29 & 23 & 22 & 14 & 5.38 & 3.61 \\
$\# 7$ & 90 & 19 & 7 & 6 & 14 & 6.57 & 4.46 \\
\hline \multirow{4}{*}{$\# 18$} & 60 & 109 & 87 & 84 & 104 & 6.75 & 6.75 \\
& 70 & 99 & 50 & 49 & 27 & 2.62 & 2.62 \\
& 90 & 89 & 62 & 60 & 26 & 2.55 & 2.55 \\
& 90 & 79 & 52 & 51 & 29 & 3.26 & 3.26 \\
\hline
\end{tabular}

We find that the predictions at starting point 60 are not good. Compared with the ARIMA model with determined $\mathrm{SOH}$, this model has better prediction results for battery \#5 and \#6 at starting points 70/80/90, and the others do not seem too bad. So when we do not have accurate $\mathrm{SOH}$ value in the real world, we can use this process to predict the RUL.

\section{Application Study with Data from the Real World}

\subsection{Data Description}

Table 6 shows some sample records of real-world data from a BMS. Each record contains the status informations of an EV at a time point, such as cell voltage, cell temperature, charge mode, mileage, and a rough SOC value. 
Table 6. Sample of real world data.

\begin{tabular}{|c|c|c|c|c|c|c|c|c|c|c|c|}
\hline vin & Time & Mode & soc & $i$ & $v_{1}$ & $\cdots$ & $t_{1}$ & $\cdots$ & lon & lat & odomileage \\
\hline test1 & 2016/9/22 20:29:01 & 2 & 61.6 & 1.64 & 3.28 & & 25 & & 0 & 0 & 192 \\
\hline test1 & 2016/9/22 20:29:11 & 1 & 61.6 & 0 & 3.28 & & 25 & & 0 & 0 & 192 \\
\hline test1 & 2016/9/23 01:31:01 & 1 & 61.6 & 0 & 3.28 & & 25 & & 0 & 0 & 192 \\
\hline test1 & 2016/9/26 06:15:01 & 4 & 61.6 & -8.58 & 3.3 & & 26 & & 0 & 0 & 192 \\
\hline test1 & $2016 / 9 / 26$ 06:15:11 & 4 & 61.6 & -8.58 & 3.3 & & 26 & & 121.446 & 31.218 & 192 \\
\hline test1 & $2016 / 9 / 26$ 06:15:21 & 4 & 61.6 & -8.48 & 3.32 & & 26 & & 121.446 & 31.218 & 192 \\
\hline
\end{tabular}

The variable mode means charge mode; soc means the rough SOC value; $i$ means charge or discharge current; $v_{k}$ means voltage of the $k$-th battery cell; $t_{k}$ means temperature of the $k$-th battery cell; lon means longitude; lat means latitude; odomileage means ODO mileage.

We need to estimate battery capacity based on these values to calculate the $\mathrm{SOH}$. From time point $t_{1}$ (the start time of a discharge cycle) to $t_{2}$ (the end time of a discharge cycle), the total capacity of the battery cell is [44]:

$$
C=C_{1,2}=\frac{Q_{1}-Q_{2}}{S O C_{1}-S O C_{2}}=\frac{\int_{t_{1}}^{t_{2}} I(t) d t}{\operatorname{SOC}\left(t_{1}\right)-\operatorname{SOC}\left(t_{2}\right)}
$$

In the real world, the number of BMS data is huge: there are usually several records in $1 \mathrm{~s}$. Sometimes there are errors in the data, which need to be cleaned.

The data come from a BMS that contains 363 vehicles samples. The data contain real-time current, voltage, temperature, and other information, as well as a rough estimate of the SOC.

We use Equation (5) to estimate the rough value of capacity and use it to compute the $\mathrm{SOH}$ value by following the Equation (2). Figure 11 shows the $\mathrm{SOH}$ details of all the vehicles. It is a smoothed color density representation of a scatterplot, obtained through a kernel density estimate. Dark area means high density of data points.

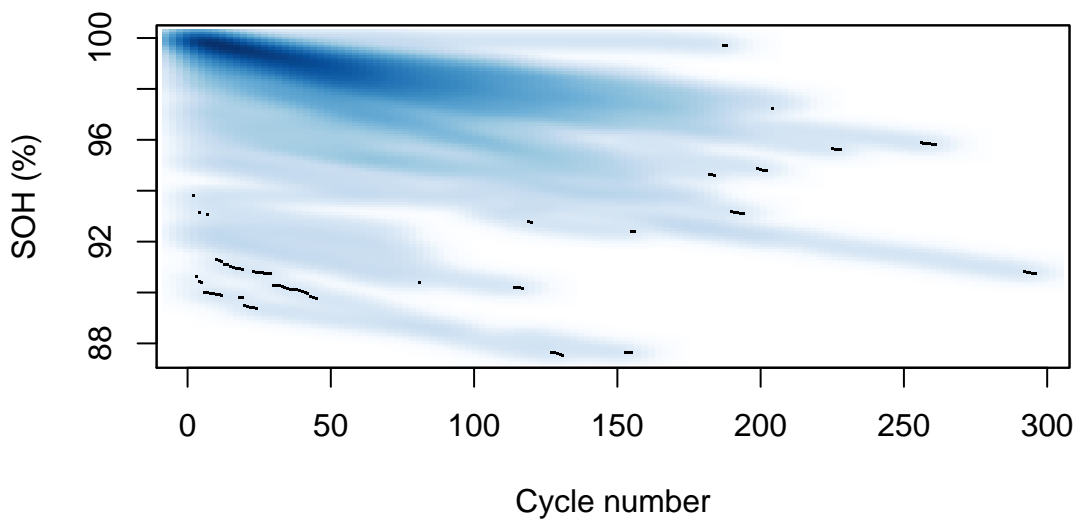

Figure 11. Real-world data-SOH based on the cycle number.

\subsection{SOH Estimation}

We found that the maximum number of cycles was only 300 , and the batteries of these vehicles are far from the EOL; therefore, there is no way to obtain exact future $\mathrm{SOH}$ values. We can use the methods mentioned in the previous section to create models with the $\mathrm{SOH}$ and various battery parameters and then use these models to predict the future $\mathrm{SOH}$. It should be noted that many vehicles do not contain enough data points, and there is a large number of vehicles in the real world; therefore, it is necessary to build an aggregate model to predict each vehicle.

As mentioned above, we continue to use the three models: the linear regression, the ridge regression, and the Lasso regression. For each method, we create an aggregate 
model (use all of the samples) and predict the vehicles separately. Table 7 shows the RMSE results.

Table 7. RMSE (\%) results of all the models.

\begin{tabular}{cccc}
\hline & Linear Regression & Ridge Regression & Lasso Regression \\
\hline Mean of RMSE & 0.92 & 0.93 & 0.92 \\
Median of RMSE & 0.6 & 0.62 & 0.61 \\
Maximum of RMSE & 8.56 & 8.6 & 8.56 \\
Minimum of RMSE & 0.08 & 0.08 & 0.08 \\
\hline
\end{tabular}

We found that there is little difference between the results of these models. The Ridge regression obtained the best performance when we use NASA dataset, so we selected the Ridge regression for the subsequent prediction.

\subsection{RUL Prediction}

To predict the future $\mathrm{SOH}$, we need the future battery parameters. In practice, we can use a Monte Carlo simulation to determine the PDF of the RUL. Without losing generality, we investigate the historical data of two features, the charge mode and mileage, for vehicle \#1. Figure 12 shows their distributions.
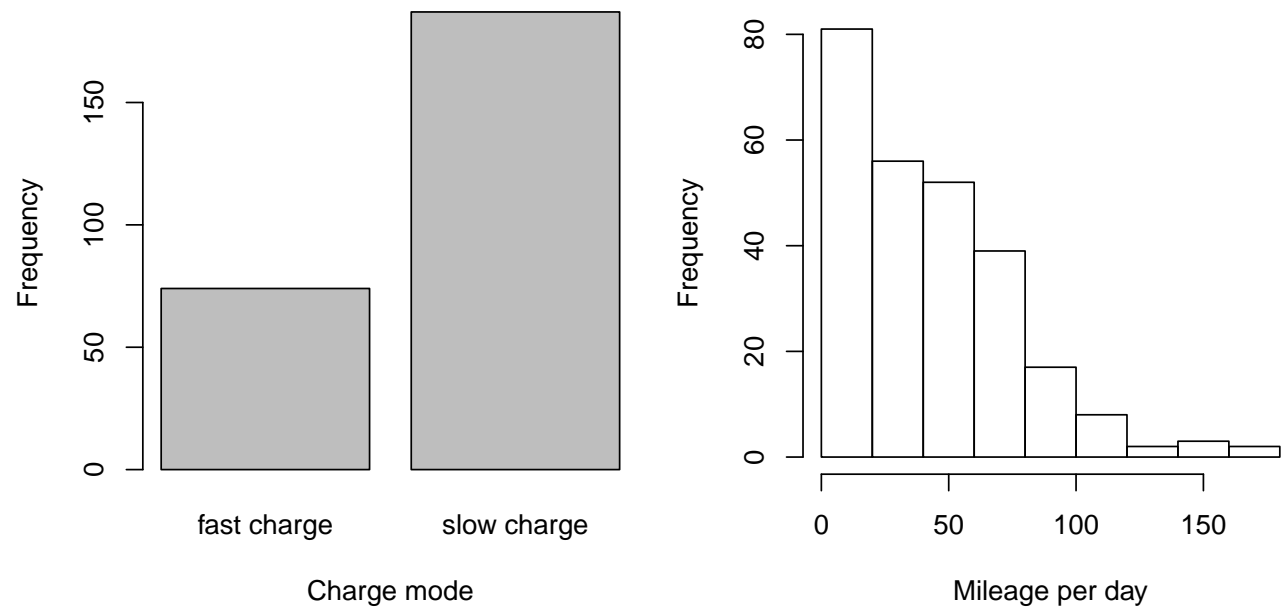

Figure 12. Frequency of the charge model and mileage per day for vehicle \#1.

We can use a binomial distribution to simulate charge mode and a log-normal distribution to simulate mileage per day. The parameters are shown in the Table 8 .

Table 8. Distribution parameters of the charge model and mileage per day for vehicle \#1.

\begin{tabular}{ccc}
\hline Feature & Distribution & Parameters \\
\hline Charge mode & binomial & $n=1, p=0.284$ \\
Mileage per day & log-normal & $\mu=3.482, \sigma=0.981$ \\
\hline
\end{tabular}

Then, future data over a period of time can be simulated. We did multiple simulations and compute the PDF of the RUL. Figure 13 is the result of four simulation scenarios for vehicle \#1; each scenario used a different random number seed. Table 9 shows the simulation parameters. Appendix A shows the R codes. 

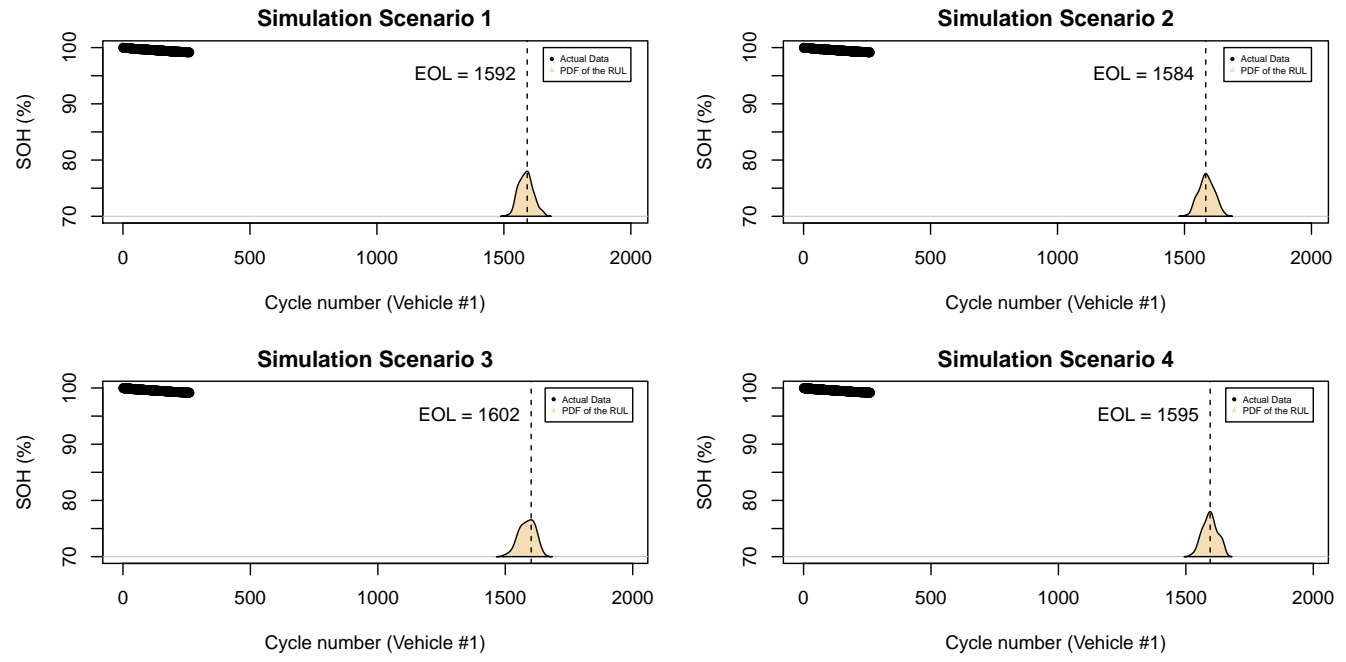

Figure 13. RUL Prediction.

Table 9. Simulation parameters for vehicle \#1.

\begin{tabular}{cccc}
\hline Scenario & Random Number Seed & Simulation Times & Maximum Cycles \\
\hline 1 & 1 & 100 & 5000 \\
2 & 2 & 100 & 5000 \\
3 & 3 & 100 & 5000 \\
4 & 4 & 100 & 5000 \\
\hline
\end{tabular}

For example, in scenario 1, according to the peak value of the density estimation, the predicted EOL for vehicle \#1 is the 1592th cycle. Since the last cycle number for prediction is 261, the RUL is 1331 cycles.

In the four simulation scenarios, the minimum of predicted EOL is 1584, the maximum of predicted EOL is 1602 , the difference rate is $1.14 \%$, and it shows that the simulated result is quite stable.

We have run 1000 simulations to compute the PDF, the total CPU time on a PC (AMD Ryzen $53500 \mathrm{U}$ ) is $0.03 \mathrm{~s}$. The computing performance can meet the daily needs.

\subsection{Discussion}

When we compute the $\mathrm{SOH}$ value of a $\mathrm{EV}$, we can predict the short-term demand for electricity. When we compute the RUL of a EV, we can predict the long-term demand for electricity. The electric demands of EVs was taken account into basic load frequency control (LFC) of power systems. LFC in power systems can generate control signals and apply it to the governors to match supply and demand. There are lots of methods to improve the performance of LFCs by tuning them such as $[45,46]$. In order to handle the parametric and non-parametric uncertainties of realistic power systems, Alhelou et al. [47] devoloped a unknown input functional observer (UIFO) [47] or unknown input observer (UIO) [48] for LFC. The unknown input observer can also be used for fault detection and isolation [49]. If we can accurately predict the electric demands, we can provide more information for LFC.

\section{Conclusions and Future Works}

A novel process for predicting the RUL using real-world data was proposed. First, the SOC-based equation was used to estimate the capacity and $\mathrm{SOH}$ of the battery for each electric vehicle. Second, a Lasso regression model was constructed with the aggregated data for all the vehicles after missing and invalid values were removed. Then, the future internal battery parameters and real-world parameters were predicted by the ARIMA model or simulated by the Monte Carlo method. Finally, the future values of the $\mathrm{SOH}$ 
were predicted by the Lasso model with the predicted parameters, and the probability distribution of the RUL was determined.

This approach is less reliant on experimental data and can easily perform real-time prediction; therefore, it is economical to apply it to evaluate a vehicle's condition and the residual value of the battery. It has significance for the battery recovery industry and thus has some contribution to the green supply chain of the automobile industry. Based on Section 3.4, we will use the RUL prediction model to predict the electric demands and make more improvement to the LFC of the power systems in future work.

Author Contributions: Conceptualization, J.L., B.-C.S. and M.C.; data curation, J.L.; formal analysis, X.W., C.-W.H.; methodology, J.L. and X.W.; project administration, B.-C.S., Y.-W.K. and M.C.; software, J.L.; supervision, M.C.; validation, Y.-W.K.; writing—original draft, J.L.; writing-review and editing, X.W., C.-W.H. and Y.-W.K. All authors have read and agreed to the published version of the manuscript.

Funding: This work was supported and funded by the Ministry of Science and Technology of Taiwan (MOST 109-2221-E-030_011_MY3).

Institutional Review Board Statement: Not applicable.

Informed Consent Statement: Not applicable.

Data Availability Statement: Available online: https:/ /ti.arc.nasa.gov/tech/dash/groups/pcoe/ prognostic-data-repository, accessed on 2 November 2021.

Conflicts of Interest: The authors declare no conflict of interest.

\section{Abbreviations}

The following abbreviations are used in this manuscript:

EV electric vehicle

BMS battery-management system

SOC state of charge

$\mathrm{SOH}$ state of health

RUL remaining useful life

EOL end of life

PDF probability density function

OVC open-circuit voltage

AIC Akaike information criterion

LFC load frequency control

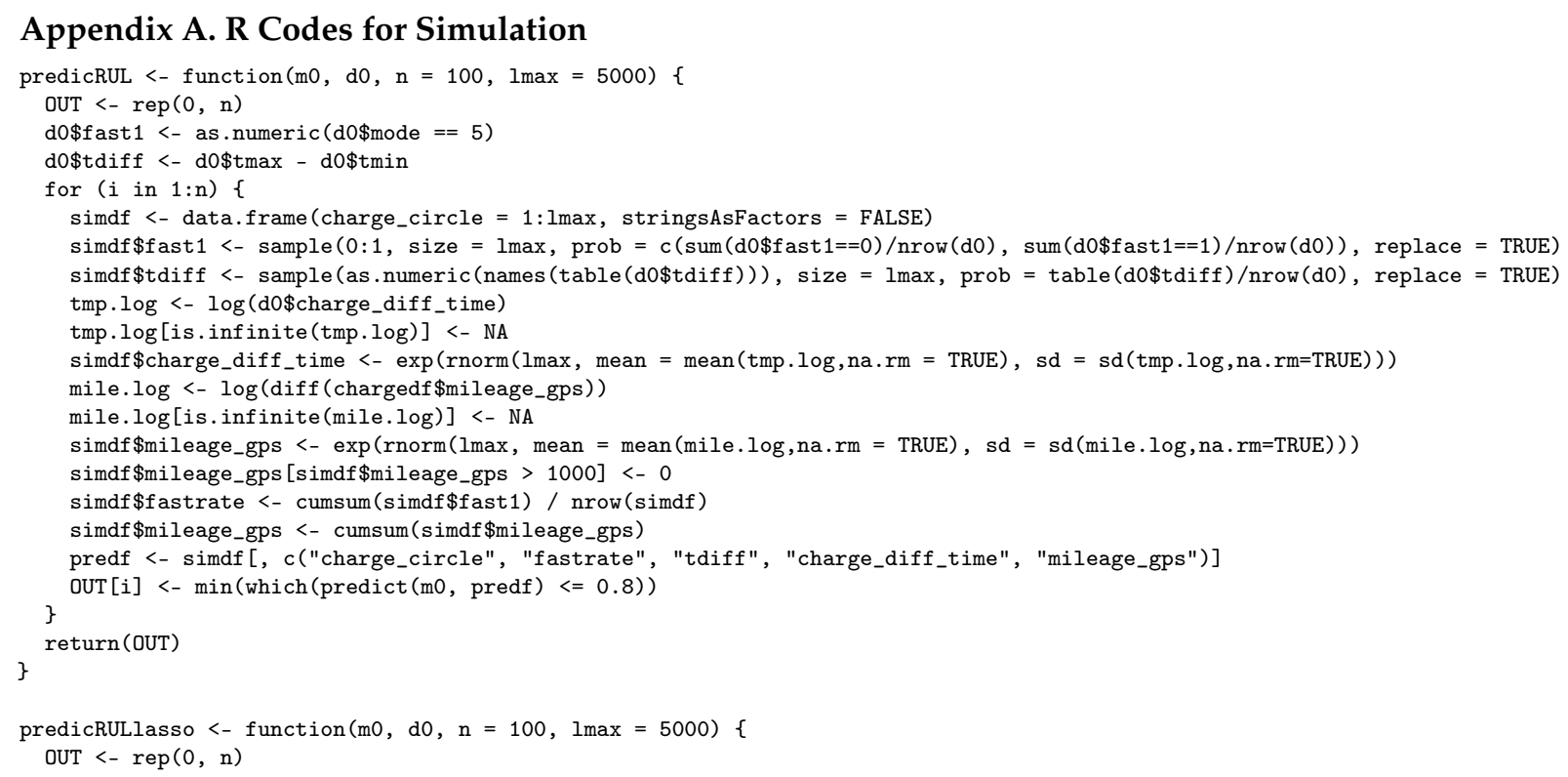




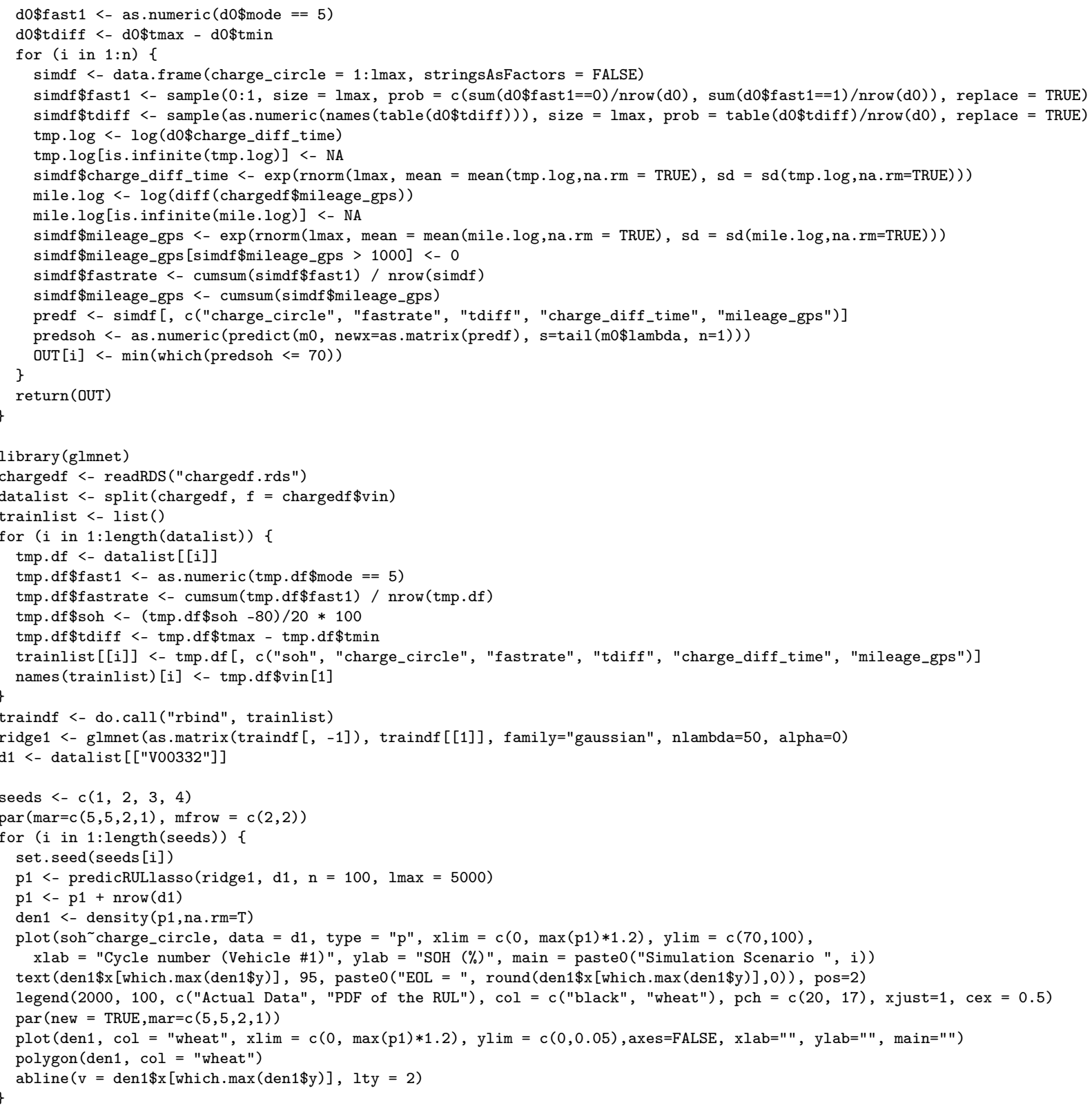

\section{References}

1. Vetter, J.; Novák, P.; Wagner, M.R.; Veit, C.; Möller, K.C.; Besenhard, J.O.; Winter, M.; Wohlfahrt-Mehrens, M.; Vogler, C.; Hammouche, A. Ageing mechanisms in lithium-ion batteries. J. Power Sources 2005, 147, 269-281. [CrossRef]

2. Xing, Y.; Ma, E.W.M.; Tsui, K.L.; Pecht, M. Battery Management Systems in Electric and Hybrid Vehicles. Energies 2011, 4, $1840-1857$. [CrossRef]

3. Pattipati, B.; Sankavaram, C.; Pattipati, K.R. System Identification and Estimation Framework for Pivotal Automotive Battery Management System Characteristics. IEEE Trans. Syst. Man Cybern. Part C 2011, 41, 869-884. [CrossRef]

4. Finger, E.P.; Marwell, E.M. Battery Control System for Battery Operated Vehicles. U.S. Patent 4,012,681, 1 September 1977.

5. Chiang, Y.H.; Sean, W.Y.; Ke, J.C. Online estimation of internal resistance and open-circuit voltage of lithium-ion batteries in electric vehicles. J. Power Sources 2011, 196, 3921-3932. [CrossRef]

6. Zhang, J.; Lee, J. A review on prognostics and health monitoring of Li-ion battery. J. Power Sources 2011, 196, 6007-6014. [CrossRef]

7. Waag, W.; Fleischer, C.; Sauer, D.U. Critical review of the methods for monitoring of lithium-ion batteries in electric and hybrid vehicles. J. Power Sources 2014, 258, 321-339. [CrossRef]

8. Wei, J.; Dong, G.; Chen, Z. Remaining Useful Life Prediction and State of Health Diagnosis for Lithium-Ion Batteries Using Particle Filter and Support Vector Regression. IEEE Trans. Ind. Electron. 2018, 65, 5634-5643. [CrossRef]

9. Zhou, Y.; Huang, M.; Chen, Y.; Tao, Y. A novel health indicator for on-line lithium-ion batteries remaining useful life prediction. J. Power Sources 2016, 321, 1-10. [CrossRef] 
10. Barré, A.; Deguilhem, B.; Grolleau, S.; Gérard, M.; Suard, F.; Riu, D. A review on lithium-ion battery ageing mechanisms and estimations for automotive applications. J. Power Sources 2013, 241, 680-689. [CrossRef]

11. Zhao, Q.; Qin, X.; Zhao, H.; Feng, W. A novel prediction method based on the support vector regression for the remaining useful life of lithium-ion batteries. Microelectron. Reliab. 2018, 85, 99-108. [CrossRef]

12. Song, Y.; Liu, D.; Yang, C.; Peng, Y. Data-driven hybrid remaining useful life estimation approach for spacecraft lithium-ion battery. Microelectron. Reliab. 2017, 75, 142-153. [CrossRef]

13. Si, X.S.; Wangbde, W.; Zhouc, D.H. Remaining useful life estimation-A review on the statistical data driven approaches. Eur. J. Oper. Res. 2011, 213, 1-14. [CrossRef]

14. Zheng, X.; Fang, H. An integrated unscented kalman filter and relevance vector regression approach for lithium-ion battery remaining useful life and short-term capacity prediction. Reliab. Eng. Syst. Saf. 2015, 144, 74-82. [CrossRef]

15. Yang, F.; Wang, D.; Xing, Y.; Tsui, K.L. Prognostics of Li(NiMnCo)O2-based lithium-ion batteries using a novel battery degradation model. Microelectron. Reliab. 2017, 70, 70-78. [CrossRef]

16. Burgess, W.L. Valve Regulated Lead Acid battery float service life estimation using a Kalman filter. J. Power Sources 2009, 191, 16-21. [CrossRef]

17. Dong, H.; Jin, X.; Lou, Y.; Wang, C. Lithium-ion battery state of health monitoring and remaining useful life prediction based on support vector regression-particle filter. J. Power Sources 2014, 271, 114-123. [CrossRef]

18. Zhang, X.; Miao, Q.; Liu, Z. Remaining useful life prediction of lithium-ion battery using an improved UPF method based on MCMC. Microelectron. Reliab. 2017, 75, 288-295. [CrossRef]

19. Duong, P.L.T.; Raghavan, N. Heuristic Kalman optimized particle filter for remaining useful life prediction of lithium-ion battery. Microelectron. Reliab. 2018, 81, 232-243. [CrossRef]

20. Zhang, H.; Miao, Q.; Zhang, X.; Liu, Z. An improved unscented particle filter approach for lithium-ion battery remaining useful life prediction. Microelectron. Reliab. 2018, 81, 288-298. [CrossRef]

21. Ma, Y.; Chen, Y.; Zhou, X.; Chen, H. Remaining Useful Life Prediction of Lithium-Ion Battery Based on Gauss-Hermite Particle Filter. IEEE Trans. Control Syst. Technol. 2019, 27, 1788-1795. [CrossRef]

22. Li, L.; Saldivar, A.A.F.; Bai, Y.; Li, Y. Battery Remaining Useful Life Prediction with Inheritance Particle Filtering. Energies 2019, 12, 2784. [CrossRef]

23. He, W.; Williard, N.; Osterman, M.; Pecht, M. Prognostics of lithium-ion batteries based on Dempster-Shafer theory and the Bayesian Monte Carlo method. J. Power Sources 2011, 196, 10314-10321. [CrossRef]

24. Ng, S.S.Y.; Xing, Y.; Tsui, K.L. A naive Bayes model for robust remaining useful life prediction of lithium-ion battery. Appl. Energy 2014, 118, 114-123. [CrossRef]

25. Patil, M.A.; Tagade, P.; Hariharan, K.S.; Kolake, S.M.; Song, T.; Yeo, T.; Doo, S. A novel multistage Support Vector Machine based approach for Li ion battery remaining useful life estimation. Appl. Energy 2015, 159, 285-297. [CrossRef]

26. Zhou, Y.; Huang, M. Lithium-ion batteries remaining useful life prediction based on a mixture of empirical mode decomposition and ARIMA model. Microelectron. Reliab. 2016, 65, 265-273. [CrossRef]

27. Zhang, Y.; Xiong, R.; He, H.; Pecht, M.G. Lithium-Ion Battery Remaining Useful Life Prediction With Box-Cox Transformation and Monte Carlo Simulation. IEEE Trans. Ind. Electron. 2019, 66, 1585-1597. [CrossRef]

28. $\mathrm{Wu}, \mathrm{J} . ; \mathrm{Zhang}, \mathrm{C} . ;$ and neural networks. Appl. Energy 2016, 173, 134-140. [CrossRef]

29. Zhang, Y.; Xiong, R.; He, H.; Pecht, M.G. Long Short-Term Memory Recurrent Neural Network for Remaining Useful Life Prediction of Lithium-Ion Batteries. IEEE Trans. Veh. Technol. 2018, 67, 5695-5705. [CrossRef]

30. Khumprom, P.; Yodo, N. A Data-Driven Predictive Prognostic Model for Lithium-ion Batteries based on a Deep Learning Algorithm. Energies 2019, 12, 660. [CrossRef]

31. Chen, L.; Tao, L.; Fan, H. Li-ion battery capacity estimation: A geometrical approach. J. Power Sources 2014, 261, $141-147$.

32. Wang, D.; Yang, F.; Zhao, Y.; Tsui, K.L. Battery remaining useful life prediction at different discharge rates. Microelectron. Reliab. 2017, 78, 212-219. [CrossRef]

33. Eddahech, A.; Briat, O.; Woirgard, E.; Vinassa, J.M. Remaining useful life prediction of lithium batteries in calendar ageing for automotive applications. Microelectron. Reliab. 2012, 52, 2438-2442. [CrossRef]

34. Nuhic, A.; Terzimehic, T.; Soczka-Guth, T.; Buchholz, M.; Dietmayer, K. Health diagnosis and remaining useful life prognostics of lithium-ion batteries using data-driven methods. J. Power Sources 2013, 239, 680-688. [CrossRef]

35. Canals, L.; Schiffer Gonzalez, A.; Amante, B.; Llorca, J. PHEV battery ageing study using voltage recovery and internal resistance from On-board data. IEEE Trans. Veh. Technol. 2015, 65, 4209-4216. [CrossRef]

36. Alhelou, H.H.; Hamedani-Golshan, M.E.; Heydarian-Forushani, E.; Al-Sumaiti, A.S.; Siano, P. Decentralized fractional order control scheme for LFC of deregulated nonlinear power systems in presence of EVs and RER. In Proceedings of the 2018 International Conference on Smart Energy Systems and Technologies (SEST), Seville, Spain, 10-12 September 2018; pp. 1-6.

37. Saha, B.; Goebel, K. Battery Data Set. In Nasa Ames Prognostics Data Repository; NASA Ames Research Center: Moffett Field, CA, USA, 2007. Available online: https:/ / ti.arc.nasa.gov/tech/dash/groups/pcoe/prognostic-data-repository (accessed on 30 November 2021).

38. Tibshirani, R. Regression Shrinkage and Selection Via the Lasso. J. R. Stat. Soc. 1996, 58, 267-288. [CrossRef] 
39. Mansouri, S.S.; Karvelis, P.; Georgoulas, G.; Nikolakopoulos, G. Remaining Useful Battery Life Prediction for UAVs based on Machine Learning. IFAC-PapersOnLine 2017, 50, 4727-4732. [CrossRef]

40. Friedman, J.; Hastie, T.; Tibshirani, R. Regularization Paths for Generalized Linear Models via Coordinate Descent. J. Stat. Softw. Artic. 2010, 33, 1-22. [CrossRef]

41. Brockwell, P.J. Time Series: Theory and Methods; Springer: New York, NY, USA, 1987.

42. Box, G.E.P.; Jenkins, G.M. Time Series Analysis : Forecasting and Control; Holden-Day: San Francisco, CA, USA, 1970.

43. Hyndman, R.; Khandakar, Y. Automatic Time Series Forecasting: The forecast Package for R. J. Stat. Softw. Artic. 2008, 27, 1-22.

44. Einhorn, M.; Conte, F.V.; Kral, C.; Fleig, J. A method for online capacity estimation of lithium ion battery cells using the state of charge and the transferred charge. IEEE Trans. Ind. Appl. 2012, 48, 736-741. [CrossRef]

45. Haes Alhelou, H.; Hamedani Golshan, M.E.; Hajiakbari Fini, M. Wind driven optimization algorithm application to load frequency control in interconnected power systems considering GRC and GDB nonlinearities. Electr. Power Compon. Syst. 2018, 46, 1223-1238. [CrossRef]

46. Alhelou, H.H.; Siano, P.; Tipaldi, M.; Iervolino, R.; Mahfoud, F. Primary Frequency Response Improvement in Interconnected Power Systems Using Electric Vehicle Virtual Power Plants. World Electr. Veh. J. 2020, 11, 40. [CrossRef]

47. Alhelou, H.H.; Golshan, M.E.H.; Hatziargyriou, N.D. A decentralized functional observer based optimal LFC considering unknown inputs, uncertainties, and cyber-attacks. IEEE Trans. Power Syst. 2019, 34, 4408-4417. [CrossRef]

48. Alhelou, H.H.; Golshan, M.E.H.; Hatziargyriou, N.D. Deterministic dynamic state estimation-based optimal lfc for interconnected power systems using unknown input observer. IEEE Trans. Smart Grid 2019, 11, 1582-1592. [CrossRef]

49. Alhelou, H.H.; Golshan, M.H.; Askari-Marnani, J. Robust sensor fault detection and isolation scheme for interconnected smart power systems in presence of RER and EVs using unknown input observer. Int. J. Electr. Power Energy Syst. 2018, 99, 682-694. [CrossRef] 